\title{
Reinforcement effect of cement grout on soil based on granular flow theory
}

\author{
Junhua Zhou ${ }^{1,2}$ Kai Fang $^{1,2, a}$ Tongbin Zhao ${ }^{1,2}$ \\ ${ }^{1}$ State Key Laboratory of Mining Disaster Prevention and Control Co-founded by Shandong \\ Province and the Ministry of Science and Technology, Shandong University of Science and \\ Technology, Qingdao, 266590. China; \\ ${ }^{2}$ Department of Mechanics, Shandong University of Science and Technology. \\ azjufangkai@163.com
}

Keywords: grouted soil, reinforcement effect, stress strain relationship, PFC2D.

\begin{abstract}
Reinforcement effect of cement grout injection on soil was studied using PFC2D numerical simulation test. The effect of cement particle size distribution, soil particle size distribution and the parallel bond strength between particles on mechanical properties of cement soil after reinforcement was analyzed. The stress-strain curve and the ultimate bearing capacity of cement grouting soil were studied by the numerical tests. The results show that under the same amount of cement, the cemented soil with small cement particle size shows a higher ultimate stress and a larger strain corresponding to the ultimate stress. The ultimate stress increases slowly with the decreasing particle size under a certain range. Ultimate stress of the cemented soil increases with the increase of the parallel bond strength. In addition, the cemented soil with large parallel bond strength shows a larger strain corresponding to the ultimate stress.
\end{abstract}

\section{Introduction}

Post grouting technology has been employed throughout the world as a routine construction process because of its technical and economical advantages. A large number of engineering practices prove that grouting at the pile tip can effectively increase the pile side frictional resistance and end resistance with the effect of penetration and compaction of the grout. Research into the reinforcement mechanism of the cement grout is of great important for the application of the post grouting technology.

Discrete Element Method, which was proposed by Cundall in 1971, was a valuable and effective technique to study geotechnical problems[1]. In 1978, Cundall and Strack proved that the discrete element method was an effective tool to study the constitutive relation of granular media[2]. Particle flow theory[3], as a special kind of discrete element method, was widely applied to study the mechanical behavior of granular media in many fields of geotechnical engineering in recent years[4]. Huang[5] studied the similarity relationship between the microscopic parameters and the macroscopic parameters of the viscous granular materials using two-dimensional particle flow program. Zhang Hongwu etc.[6] calibrated the micro parameters of the numerical analysis model of the granular flow based on the stress-strain results obtained from the indoor triaxial test. Particle flow numerical simulation method can be used to study the properties of particles by simulating the interaction between the particles and the motion of the particles. PFC, which is one kind of the particle flow numerical simulation software, can be convenient to deal with non-continuum mechanics problem, reflecting the multiphase medium of different physical relationship. It can effectively simulate the process of grout injection into soil and be used to study the soil reinforcement mechanism.

In this paper, the uniaxial compression test of grouting cement soil was simulated by particle flow software PFC2D. The mechanism reinforcement of cement grout injection on soil was studied. And the corresponding influencing factors were also analyzed. 


\section{Numerical analysis}

Numerical model. The soil sample, with a size of $100 \mathrm{~mm} \times 50 \mathrm{~mm}$ was simulated by a series of particles. Radius of the particle distributed from $\mathrm{R}_{\min }$ to $\mathrm{R}_{\max }$ randomly. The initial porosity of the soil sample is 0.3 . The porosity decreases to 0.1 after the injection of cement grout. The basic parameters of numerical test are shown in Table 1.

Table 1 Microscopic parameters of the model

\begin{tabular}{ccc}
\hline Mechanical parameters & Cohesive soil particles & Cement particles \\
\hline $\mathrm{R}_{\min }(\mathrm{mm})$ & 0.5 & 0.13 \\
$\mathrm{R}_{\max } / \mathrm{R}_{\min }$ & 2.7 & 1.5 \\
Density $\left({\left.\mathrm{Kg} \times \mathrm{m}^{-3}\right)}^{\text {Friction coefficient }}\right.$ & 1650 & 2750 \\
Normal stiffness $(\mathrm{Pa})$ & 0.7 & 0.7 \\
Stiffness ratio & $2.1 \times 107$ & $1.8 \times 108$ \\
Parallel bond tensile strength $(\mathrm{Pa})$ & 2.5 & 2.5 \\
Parallel bond strength ratio & $4.7 \times 103$ & $5 \times 104$ \\
Parallel bond stiffness ratio & 1 & 1 \\
Parallel bond radius factor & 2.5 & 2.5 \\
\hline
\end{tabular}

4 walls with a range of $100 \mathrm{~mm} \times 50 \mathrm{~mm}$ were defined firstly. Particles of a given radius were randomly generated in the region. The confining pressure and loading process of the test were realized by using the PFC wall. Due to the existence of the wall, the boundary effect can occur during the experiment. In order to eliminate the effect between the wall and the particles, the coefficient of friction between the particles and the particles was chosen as the same with the friction coefficient between the particles. The model is shown in Figure 1. It is clearly seen that the cement particles (red) are bonded with soil particles (blue).
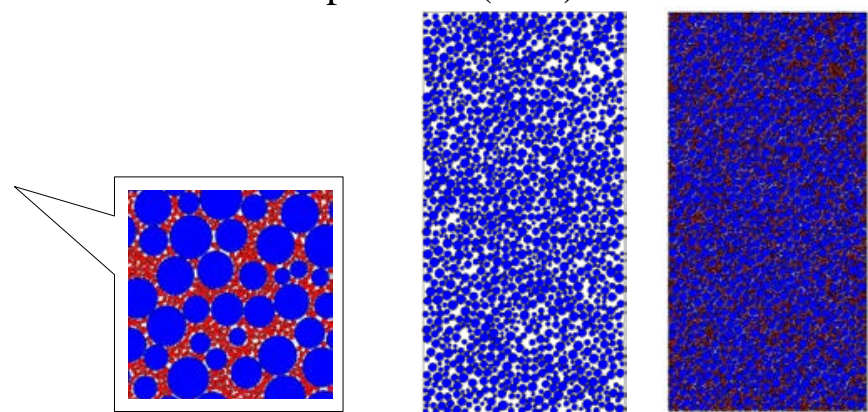

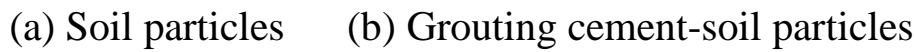

Fig. 1 PFC2D numerical model

Tests results. In order to evaluate the effect of grouting on the bearing behavior of pile, PFC numerical simulation was conducted to study the reinforcement of the grout. The effect of cement particle size distribution, soil particle size distribution and the parallel bond strength between particles on mechanical properties of cement soil after reinforcement were analyzed. The numerical simulation parameters of the simulation test are shown in Table 2.

Table 2 PFC numerical simulation parameters

\begin{tabular}{ccc}
\hline Cement particle size $(\mathrm{mm})$ & Soil particle size $(\mathrm{mm})$ & Parallel bond strength $(\mathrm{KPa})$ \\
\hline $0.13-0.20$ & $0.40-1.35$ & 13.0 \\
$0.14-0.21$ & $0.45-1.35$ & 23.0 \\
$0.15-0.22$ & $0.50-1.35$ & 33.0 \\
$0.16-0.23$ & $0.55-1.35$ & 43.0 \\
$0.17-0.24$ & $0.60-1.35$ & 53.0 \\
\hline
\end{tabular}

During the loading process, the two sides of the sample were not restricted. The loading speed of the loading plate was $0.1 \mathrm{~mm} / \mathrm{s}$. The actual unconfined condition was simulated until the failure of the sample. The corresponding stress-strain curves were obtained. 
Figure 2 was the stress-strain curves of cemented soil with different sizes of cement particles in the numerical test. It can be found from the figure that under the same amount of cement, the cemented soil with small cement particle size shows a higher ultimate stress. When the cement particle size decreases to a certain value, it has little influence on the ultimate stress. In addition, the cemented soil with small cement particle size shows a larger strain corresponding to the ultimate stress.

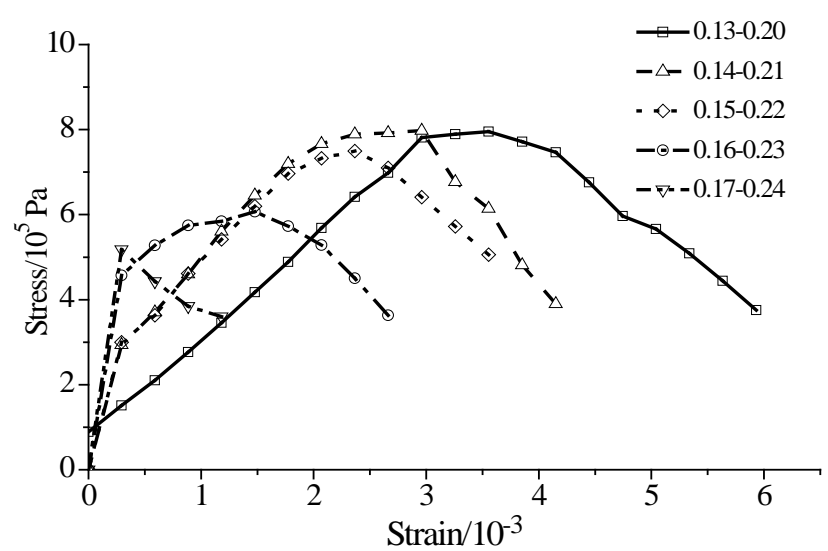

Fig. 2 Stress-strain curves of cement soil with different cement particle sizes

Figure 3 was stress-strain curves of cemented soil with different sizes of cement particles in the numerical test. Under the condition of the same amount of cement, it can be found that the effect of soil particle size on the ultimate stress of cement soil is small. For a certain range, the ultimate stress increase slowly with the decreasing particle size.

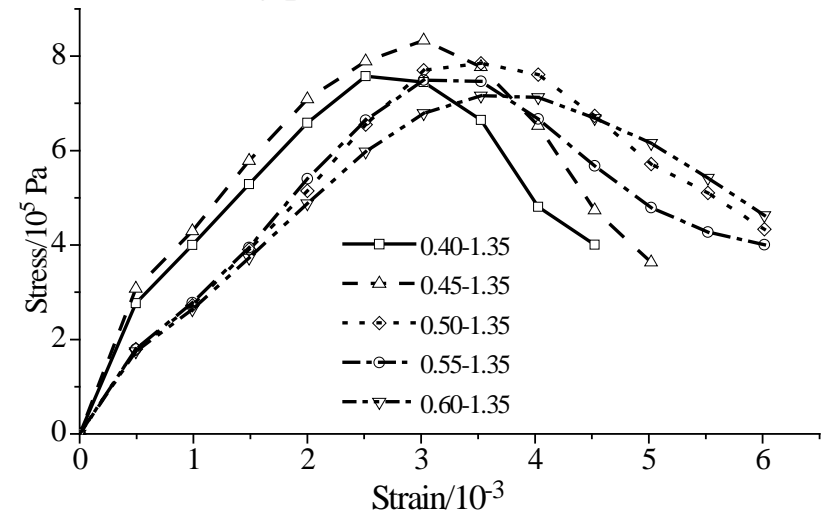

Fig. 3 Stress-strain curves of cement soil with different soil particle size

Figure 4 was the stress-strain curves of cemented soil with different parallel bond strength between soil particles and cement particles. It shows that ultimate stress of the cemented soil increases with the increase of the parallel bond strength. In addition, the cemented soil with large parallel bond strength shows a larger strain corresponding to the ultimate stress.

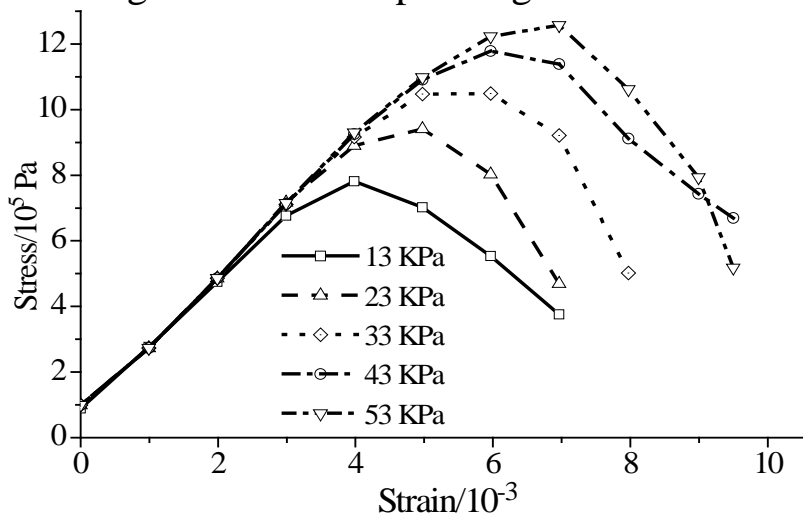

Fig. 4 Stress-strain curves of cemented soil with different parallel bond strength 


\section{Conclusions}

Reinforcement effect of cement grout injection on soil was studied using PFC2D numerical simulation test. The following conclusions are obtained:

(1) Under the same amount of cement, the cemented soil with small cement particle size shows a higher ultimate stress. In addition, the cemented soil with small cement particle size shows a larger strain corresponding to the ultimate stress;

(2) Under the condition of the same amount of cement, the effect of soil particle size on the ultimate stress of cement soil is small. For a certain range, the ultimate stress increase slowly with the decreasing particle size;

(3) Ultimate stress of the cemented soil increases with the increase of the parallel bond strength. In addition, the cemented soil with large parallel bond strength shows a larger strain corresponding to the ultimate stress.

\section{Acknowledgements}

This work is supported by National Natual Science Foundation of China (51408345), Shandong Province Natural Science Fund (No. BS2014SF012), Scientific Research Foundation of Shandong University of Science and Technology for Recruited Talents (2014RCJJ026).

\section{Reference}

[1] P. Kulatilake, H. Ucpirti, S. Wang, Use of the distinct element method to perform stress analysis in rock with non-persistent joints and to study the effect of joint geometry parameters on the strength and deformability of rock masses, Rock Mech. Rock Eng. 25 (1992) 253-274.

[2] Strack, O. D. L, P. A. Cundall, The distinct element method as a tool for research in granular media, UMN, 1978.

[3] P. A. Cundall, A discontinuous future for numerical modelling in geomechanics, Pro. Inst. Civ. Eng. Geotech. Eng. 149 (2001) 41-47.

[4] J. W. Park, J. J. Song, Numerical simulation of a direct shear test on a rock joint using a bonded-particle model, Int. J. Rock Mech. Min. Sci. 46 (2009) 1315-1328.

[5] H. Huang, Discrete element modeling of tool-rock interaction, 1999.

[6] H. Zhang, J. Qin, Simulation of mechanical behaviors of granular materials by discrete element method based on mesoscale nonlinear contact law, Chin. J. Geotech. Eng. 28 (2006) 1964-1969. 